\title{
Uso de Práticas Ágeis para Alcançar o CMMI 5: Uma Abordagem Inovadora
}

\author{
Ana Sofia C. Marçal ${ }^{1}$, Carla Ilane Moreira Bezerra ${ }^{1}$, Ciro Coelho ${ }^{2}$, Carlo Giovano \\ da Silva Pires ${ }^{1}$, Gabriela Telles de Souza ${ }^{1}$, Luciana Trindade ${ }^{1}$ \\ ${ }^{1}$ Instituto Atlântico \\ Rua Chico Lemos, 946 - Cidade dos Funcionários - Fortaleza - Ceará - Brasil \\ ${ }^{2}$ Faculdade 7 de Setembro (FA7) \\ Rua Alm. Maximiniano da Fonseca, 1395 - Fortaleza - Ceará - Brasil \\ \{marcal_sofia, carlailane, cgiovano, gabi, luciana\} datlantico.com.br; \\ ccoelho@gmail.com
}

\begin{abstract}
This paper describes how a software research and development organization implemented agile practices in adherence to CMMI for achieving a high process maturity level. This implementation has followed a strategy compliant to the Organizational Innovation and Deployment process area of CMMI using the Six Sigma's DMADV method. The process for selecting and validating the agile practices that aggregate more value and the integration of these practices in the organization's standard process are described, as well as the improvement results achieve in performance baselines.
\end{abstract}

Resumo. Este artigo descreve como uma organização de pesquisa e desenvolvimento de software introduziu práticas ágeis aderentes ao CMMI para alcançar altos níveis de maturidade de processo. Esta implementação foi realizada de acordo com a área de processo Inovação e Desenvolvimento Organizacional e utilizou o método DMADV definido pela metodologia Six Sigma. São descritos o processo de seleção e validação das práticas ágeis com melhor valor agregado e a integração dessas práticas no processo padrão da organização, bem como os resultados de melhoria alcançados nas baselines de desempenho.

\section{Introdução}

A crescente demanda por produtos de software com alto grau de atendimento aos requisitos do cliente e que apresentem melhores resultados em termos de prazo, custo e qualidade dos produtos/serviços tem motivado organizações do mundo inteiro a adotarem modelos de qualidade como o CMMI [Chrissis et al. 2006] em seus processos.

Entretanto, o surgimento de vários métodos ágeis no final dos anos 90 contribuiu para uma tendência de desenvolvimento ágil de aplicações de forma que, nos últimos anos, as organizações têm enfrentado um grande desafio para a integração entre métodos ágeis e modelos de maturidade. As organizações buscam os benefícios da abordagem 
ágil garantindo, ao mesmo tempo, a aderência ao CMMI. Neste sentido, diversos estudos e esforços têm sido feitos na tentativa de combinar práticas ágeis e CMMI.

Este artigo apresenta a experiência do Atlântico na implantação de praticas ágeis visando alcançar o nível 5 de maturidade do CMMI. Esta implantação foi realizada de acordo com a área de processo Inovação e Desenvolvimento Organizacional e com o apoio da metodologia Six Sigma [Tayntor 2003]. Um dos aspectos fundamentais deste relato é que a experiência reporta casos e resultados quantitativos considerando o contexto organizacional de alta maturidade do Atlântico, o qual inclui baselines de desempenho, gerenciamento quantitativo de projetos e inovações incorporadas de forma controlada ao processo padrão da organização.

O artigo está organizado da seguinte forma: inicialmente é apresentada uma visão geral do CMMI, explorando os altos níveis de maturidade, bem como uma breve contextualização dos métodos ágeis. A seguir é descrita a experiência do uso de práticas ágeis para alcançar o CMMI 5, incluindo a seleção das práticas ágeis, integração dessas práticas no processo padrão da organização e a análise realizada para seleção das práticas com maiorr valor agregado. Por fim serão descritos os resultados e as melhorias alcançadas, desafios, benefícios e conclusões.

\section{Enfoques sobre CMMI e Agilidade}

\subsection{Alta Maturidade no CMMI}

O Capability Maturity Model Integration (CMMI) [Chrissis et al. 2006] é um modelo de maturidade para desenvolvimento de produtos desenvolvido pelo Software Engineering Institute (SEI) que está cada vez mais sendo adotado nas empresas, uma vez que este modelo busca orientar as organizações na implementação de melhorias contínuas em seus processos de desenvolvimento. O CMMI oferece duas abordagens para melhoria de processos: a representação contínua e a representação por estágios. $\mathrm{O}$ foco deste artigo está na representação por estágios a qual define 5 níveis de maturidade organizacionais: 1 - Inicial; 2 - Gerenciado; 3 - Definido; 4 - Gerenciado Quantitativamente; e 5 Otimizado.

A alta maturidade no CMMI é alcançada nos níveis 4 e 5 da representação por estágios. No nível 4 são selecionados os sub-processos que contribuem significativamente para o desempenho geral do processo. A qualidade e o desempenho do processo são entendidos em termos estatísticos e são gerenciados durante toda a vida dos processos. Medidas de qualidade e desempenho de processos são incorporadas ao repositório de medições da organização para dar suporte a futuras decisões. Já no nível 5 os processos são continuamente melhorados com base em um entendimento quantitativo das causas comuns de variações inerentes aos processos.

Nestes dois níveis são tratadas quatro áreas de processo, das quais duas são foco deste trabalho: Desempenho do Processo Organizacional (nível 4) e Inovação e Desenvolvimento Organizacional (nível 5).

O objetivo principal da área de processo Desempenho do Processo Organizacional é estabelecer e manter um entendimento quantitativo do desempenho do conjunto de processos padrão da organização, e prover dados de desempenho de 
processo, baselines e modelos para gerência quantitativa de projetos de forma que estes alcancem os objetivos de qualidade e de desempenho esperados [Chrissis et al. 2006].

A área de processo Inovação e Desenvolvimento Organizacional permite a seleção e implementação de melhorias que possam aumentar a habilidade de uma organização em atingir seus objetivos de qualidade e de desempenho de processo. A conquista desses objetivos depende do estabelecimento bem sucedido de uma infraestrutura que permita e encoraje todas as pessoas de uma organização a propor potenciais melhorias aos seus processos e tecnologias. Projetos-piloto são conduzidos para avaliar mudanças significativas que envolvam melhorias inéditas, de alto-risco ou inovadoras, antes que sejam amplamente disseminadas [Chrissis et al. 2006].

Planejar a evolução e inovação dos processos requer uma metodologia capaz de traduzir objetivos e necessidades de negócios em metas de desempenho de processos e estas, por sua vez, em planos táticos de evolução [Hehn 2008]. Inovações nos processos precisam atender às necessidades do negócio e a satisfação do cliente utilizando metas e objetivos que possam ser mensurados. Neste contexto de inovação, o método DMADV (Define, Measure, Analyze, Design e Verify) é utilizado para implantação do Design for Six Sigma (DFSS) [Tayntor 2003] em projetos cujo escopo é o desenvolvimento de novos produtos, processos e serviços. O DMADV proporciona inovação ao processo focando o fornecimento de valor através da criação de um novo produto ou serviço e do desenvolvimento de novos processos de trabalho [Watson 2007].

\subsection{Métodos Ágeis para Desenvolvimento de Software}

Aparentemente opostos aos modelos padrões para o desenvolvimento de software, os métodos ágeis surgiram em meio à crise crônica do software como uma reação aos modelos clássicos e tradicionais de desenvolvimento e do reconhecimento da necessidade de se criar uma alternativa a estes processos caracterizados pelo grande foco dado à criação de documentações completas [Beck et al. 2001]. O surgimento de vários métodos ágeis no final dos anos 90, tais como eXtreme Programming (XP) [Beck 1999], Feature Driven Development, Modelagem Ágil [Ambler 2002] e Scrum [Schwaber 2004], contribuiu para que a partir de 2000, de acordo com Boehm [Boehm 2006], acompanhássemos uma tendência para o desenvolvimento ágil de aplicações.

Alinhado aos princípios ágeis, o Scrum foi criado em 1996 por Ken Schwaber e Jeff Sutherland, como um método que aceita que o desenvolvimento de software é imprevisível, sendo aplicável a ambientes voláteis. O Scrum se destaca dos demais métodos ágeis pela ênfase dada ao gerenciamento do projeto. Há atividades de monitoramento e feedback, em geral através de reuniões rápidas e diárias com toda a equipe, visando à identificação e correção de deficiências e/ou impedimentos no desenvolvimento. $\mathrm{O}$ método baseia-se ainda em princípios como: equipes pequenas de, no máximo, 7 pessoas; requisitos pouco estáveis ou desconhecidos; iterações curtas. Ele divide o desenvolvimento em períodos de no máximo 4 semanas, denominados sprints.

O eXtreme Programming (XP) foi criado por Kent Beck, Ron Jeffries e Ward Cunningham. O XP foi criado para projetos de desenvolvimento de software com times pequenos ou médios os quais tem requisitos vagos, pouco definidos, que mudam rapidamente ou são muito críticos. O XP baseia-se em 12 práticas ou regras concisas e diretas, a saber: jogo do planejamento; entregas frequentes; metáfora; projeto simples; 
testes antes da codificação; refactoring; programação em pares; propriedade coletiva; integração contínua; semana de 40 horas; cliente presente; e padronização do código.

A modelagem ágil, criada por Scott Ambler, corresponde a um conjunto de valores, princípios e práticas para modelagem e documentação de software. A modelagem ágil busca aplicar boas práticas para modelar sistemas de forma efetiva e ágil. Entre as características da modelagem ágil, pode-se citar o uso de modelos e ferramentas simples e a utilização de modelos descartáveis que são utilizados apenas para entendimento e não para documentação, não precisando ser mantidos atualizados nas fases posteriores do desenvolvimento.

\section{Uso de Práticas Ágeis para Alcançar o CMMI 5}

\subsection{Alta Maturidade no Atlântico}

O Instituto Atlântico é uma instituição de pesquisa e desenvolvimento localizada em Fortaleza, Ceará, com filiais em Sobral e São Paulo, e conta com aproximadamente 270 colaboradores. Desde a sua fundação, o Atlântico iniciou um amplo programa de qualidade, sendo aderente à norma ISO 9001:2008 e tendo obtido, em agosto de 2009, o nível 5 de maturidade no modelo CMMI-DEV 1.2. O Atlântico utiliza a metodologia Six Sigma para implementar os altos níveis de maturidade do CMMI. A gestão quantitativa dos processos e projetos do nível 4 do CMMI é atendida pelo controle estatístico proporcionado pelo Six Sigma, enquanto a melhoria contínua pode ser atingida pela implementação dos projetos DMAIC (Define, Measure, Analyse, Improve and Control) e a inovação que pode ser coberta por outra metodologia do Six Sigma, o DMADV (Define, Measure, Analyse, Design and Verify), atingindo assim as práticas do nível 5. Assim, projetos de melhoria DMAIC são executados com a finalidade de melhorar os processos organizacionais e os projetos de inovação DMADV são executados para implantação de uma nova tecnologia ou de novos processos.

No Atlântico, a gestão quantitativa de projetos é realizada por meio de baselines de desempenho que determinam a situação atual da organização. As baselines também fornecem a base para identificação e medição de melhorias e inovações, ajudam a determinar metas organizacionais e de projetos e auxiliam no acompanhamento do desempenho dos projetos.
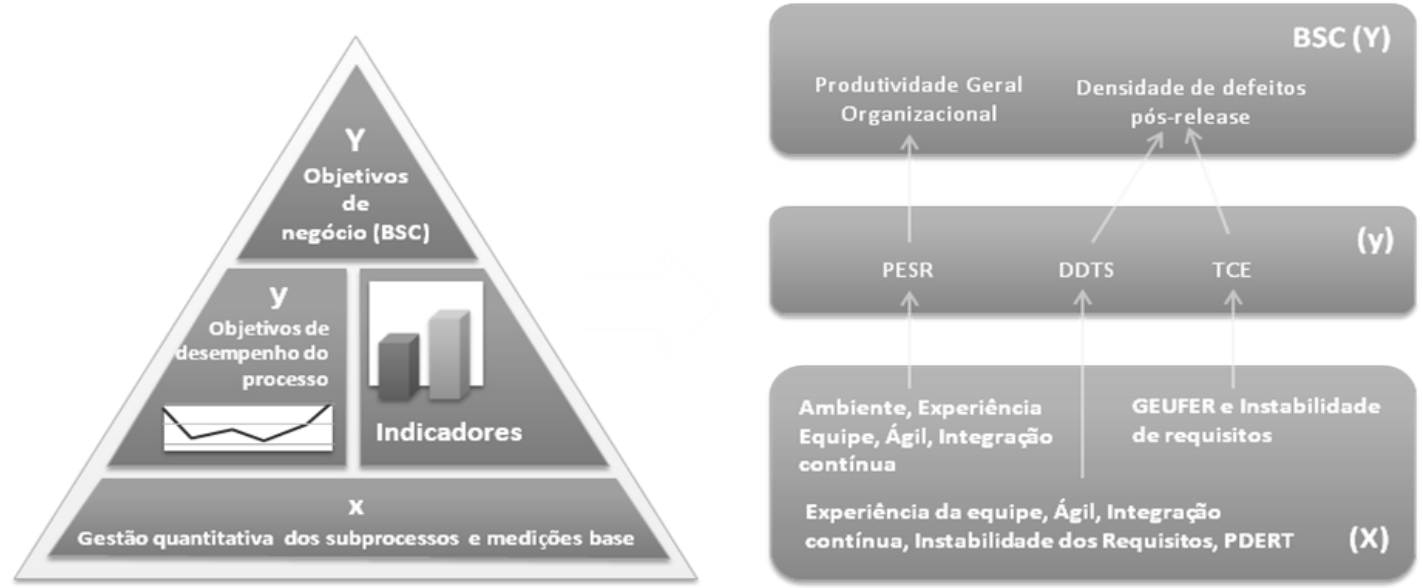

Figura 1. Baselines Organizacionais 
As baselines de desempenho do Atlântico se relacionam com os indicadores estratégicos e são representadas pelos fatores Ys e Xs como pode ser visto na Figura 1. Os fatores Ys são compostos por fatores que influenciam os Xs. Os fatores Ys são representados pela produtividade de engenharia sem retrabalho (PERS), densidade de defeitos em testes sistêmicos (DDTS) e eficácia dos testes sistêmicos (TCE). A organização também possui dois indicadores que monitoram os objetivos estratégicos da organização: a produtividade geral organizacional e a densidade de defeitos pós-release, onde este último mede a densidade de defeitos repassados para o cliente.

\subsection{Projeto DMADV Processos Ágeis}

O projeto de inovação DMADV Processos Ágeis foi iniciado a partir de uma necessidade identificada no planejamento estratégico anual da organização, tendo sido uma das ações priorizadas. O principal objetivo deste projeto foi melhorar a produtividade dos projetos de desenvolvimento de software por meio da adoção de um processo mais enxuto e que, ao mesmo tempo, fosse aderente ao CMMI. O projeto seguiu as fases Definir, Medir, Analisar, Projetar e Verificar como descritas a seguir.

$\mathrm{Na}$ fase Definir foi estabelecida, a partir da baseline de desempenho da organização, uma meta de melhoria de produtividade. A meta inicial do projeto de inovação DMADV foi definida em 15 a 20\% de melhoria da produtividade média dos projetos de software. Nessa fase foram também levantadas práticas ágeis com potencial para melhorar a produtividade e definidos os aspectos críticos que devem ser atendidos pelas soluções, os CTQs (Critical to Quality). Os CTQs adotados no projeto foram:

- Facilidade de implantação do processo;

- Impacto no esforço dos projetos;

- Impacto no modelo de produtividade;

- $\quad$ Facilidade de aderência ao CMMI;

- Impacto na qualidade do processo;

- Impacto na qualidade do produto.

$\mathrm{Na}$ fase Medir, foram coletados os dados da situação da organização e dos principais fatores que influenciam a produtividade. Também foram analisados os subprocessos ligados a esses fatores e as melhorias necessárias nos mesmos para alcançar os objetivos estabelecidos e foram detalhados os CTQs no plano de medição do projeto de inovação.

Esses dados serviram de base para a identificação, na fase Analisar, das soluções com maior retorno do investimento, representado, nesse caso, pelo potencial de melhoria da produtividade. Foram analisadas e priorizadas práticas de diversas metodologias, como XP, Scrum e modelagem ágil. A priorização dessas práticas considerou o potencial de contribuição de cada uma para a meta principal do projeto (aumento de produtividade), bem como o atendimento das soluções aos demais CTQs.

Após diversas análises estatísticas e pesquisas com participantes de projetos utilizando técnicas de análise e tomada de decisão, foram priorizadas, para implantação 
em um primeiro momento, práticas ágeis de análise e projeto, gestão de projetos e gestão de requisitos.

$\mathrm{Na}$ fase Projetar, as práticas selecionadas foram detalhadas e foi definido como as mesmas seriam integradas ao processo padrão da empresa, mantendo a aderência ao CMMI. Foram criados critérios de adaptação do processo padrão para que cada projeto pudesse escolher as práticas ágeis mais adequadas para o seu contexto específico. Em suma, criou-se uma versão ágil do processo padrão da organização.

As principais práticas adotadas podem ser vistas no Quadro 1. As práticas de análise e projeto seguem os princípios da modelagem ágil e a prática de projeto simples do XP. Já as práticas de gestão de projetos e gestão de requisitos seguem o processo definido pelo Scrummi [Marçal 2009], adaptação do Scrum aderente ao CMMI.

Quadro 1. Práticas ágeis adotadas pela organização

\begin{tabular}{|l|l|l|}
\hline Prática & Subprocesso & Prática de referência \\
\hline Iterações curtas, timebox & Gestão de Projetos & Scrum, XP \\
\hline Reuniões diárias & Gestão de Projetos & Scrum \\
\hline Estimativas realizadas pela equipe no início da iteração & Gestão de Projetos & Scrum \\
\hline $\begin{array}{l}\text { Backlog de requisitos priorizado pelo cliente, backlog da } \\
\text { iteração acordado entre cliente e equipe }\end{array}$ & Requisitos & Scrum \\
\hline $\begin{array}{l}\text { Apresentação para o cliente da versão gerada ao final de } \\
\text { cada iteração }\end{array}$ & Requisitos & Scrum \\
\hline $\begin{array}{l}\text { Modelagem ágil: modelos mais simples, modelos } \\
\text { descartáveis etc. }\end{array}$ & Análise e Projeto & XP, Modelagem Ágil \\
\hline
\end{tabular}

Essas soluções foram avaliadas em projetos-piloto, para comprovação dos resultados e alcance das metas inicialmente definidas. O primeiro projeto piloto realizou o desenvolvimento de um sistema de Gestão de Suprimentos para um cliente da indústria têxtil situado no estado do Ceará. O projeto foi iniciado em agosto de 2008 teve duração de 7 meses. Todo o projeto foi realizado incremental e iterativamente, com iterações de 4 semanas e entregas de funcionalidades ao final de cada iteração. $\mathrm{O}$ projeto possuía requisitos extremamente voláteis os quais eram definidos ao longo do projeto com grande envolvimento do cliente. O cliente participava ativamente da construção do backlog de requisitos e das reuniões de planejamento da iteração. Durante as reuniões de planejamento, os requisitos priorizados eram estimados em Story Points. Para manter a compatibilidade com a base histórica da organização, foi desenvolvido um modelo, obtido por meio de regressão linear, para conversão de Story Points em Use Case Points [Marçal et. al. 2009]. A produtividade obtida nesse projeto, no período em que foi realizado o piloto, foi $38 \%$ melhor que a produtividade planejada para o projeto.

Outro projeto piloto utilizou processo semelhante, porém em um contexto de desenvolvimento de aplicações para dispositivos móveis para uma empresa multinacional. Nesse projeto, a paricipação do cliente não foi tão efetiva, já que o mesmo encontrava-se geograficamente distante da equipe de desenvolvimento. A produtividade do projeto, no período em que foi piloto, entretanto, foi $50 \%$ melhor que a planejada no projeto. Esse resultado é ainda mais relevante considerando que a produtividade havia sido planejada em função de uma fase anterior do projeto, onde aplicativos semelhantes haviam sido desenvolvidos pela mesma equipe.

Os resultados alcançados nos projeto-piloto foram bem acima da meta inicial do projeto de inovação. Assim, considerando os resultados obtidos, a fase Verificar 
consistiu em incorporar as práticas ágeis ao processo da organização e liberá-las para uso pelos demais projetos. Foram então medidos os resultados dos projetos que adotaram as práticas ágeis para comprovar as melhorias observadas nos projetos-piloto. Os resultados obtidos foram positivos, tendo sido o projeto DMADV, então, encerrado.

\section{Principais Desafios e Benefícios}

Durante a execução do projeto de inovação DMADV, alguns desafios foram encontrados. Dentre esses, pode-se citar a necessidade de mudanças culturais na organização, já que não se pode obter agilidade apenas pela adoção de algumas práticas. Deve ser ressaltado também o trabalho de convencimento e repasse de conhecimento que precisou ser realizado junto a gestores e desenvolvedores da empresa, e consultores e avaliadores CMMI, já que muitas pessoas ainda desconhecem os princípios e práticas ágeis ou, quando as conhecem, não possuem o nível de aprofundamento necessário.

Apesar dos desafios, as melhorias disponibilizadas trouxeram vários benefícios para os projetos. Um dos benefícios mais significantes foi a comprovação da melhoria da produtividade em $52 \%$ na média e $64 \%$ na variação em relação aos projetos que não utilizavam práticas ágeis. Com isso, uma nova baseline de desempenho de produtividade organizacional foi gerada para projetos com ciclo de vida ágil como mostra a Figura 2. Dessa forma, os projetos que utilizam as práticas ágeis adotadas são quantitativamente gerenciados utilizando essa nova baseline.

\section{I-MR Chart of PESR by Baseline}

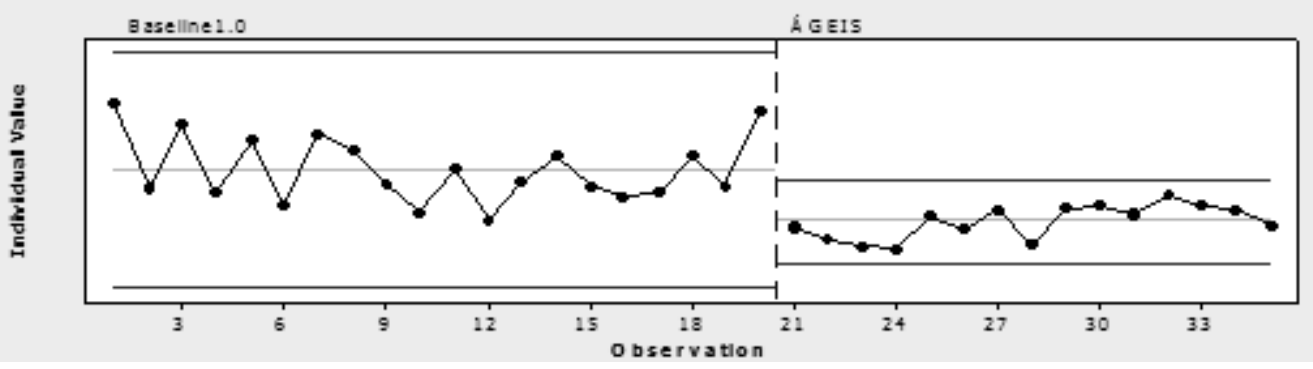

Figura 2. Baseline de Produtividade para Projetos com Ciclo de Vida Ágil

Outro benefício a ser citado foi a introdução de uma cultura de metodologias ágeis naorganização, refletida na criação de um indicador ágil para os projetos. Este indicador mede a utilização de práticas ágeis para cada projeto e é calibrado utilizandose de fatores (backlog priorizado pelo cliente, modelagem ágil e iterações timebox), pesos e fórmula de ponderação. A calibração é realizada de acordo com o uso que o projeto faz de cada um desses fatores, o que é definido de acordo com as características e necessidades do projeto. $\mathrm{O}$ indicador ágil foi incluído no modelo de desempenho de produtividade da organização, gerado com $\mathrm{R}-\mathrm{Sq}(\operatorname{adj})=74,8 \%$ e equação:

\section{Produtividade (PERS) = 36,3 - 2,15 Ambiente - 6,35 Grau de Utilização de Integração Contínua - 0,255 Experiência da Equipe - 1,02 Ágil}

$\mathrm{O}$ fator de produtividade (PERS) representa o esforço necessário para implementar uma unidade de tamanho, no caso, Pontos de Caso de Uso. Assim, quanto menor o PERS, melhor a produtividade. De acordo com o modelo, quanto maior é o 
fator ágil melhor é a produtividade, comprovando que as práticas ágeis realmente influenciam positivamente a produtividade.

\section{Conclusões}

Este trabalho apresenta uma experiência de adoção de práticas de desenvolvimento ágil em compatibilidade com o nível 5 do modelo CMMI. As práticas ágeis foram implantadas na organização como parte de um projeto de inovação DMADV, ferramenta para inovação prevista pela metodologia Six Sigma.

O principal objetivo da adoção de práticas ágeis foi a melhoria da produtividade dos projetos de desenvolvimento de software, tendo esse objetivo sido não só atingido, mas largamente ultrapassado.

Além disso, projetos que utilizaram essas práticas foram submetidos, com sucesso, a avaliações oficiais CMMI. Assim, pode-se comprovar a viabilidade do uso de métodos ágeis de forma aderente ao CMMI.

Apesar dos desafios, conclui-se que os benefícios excedem em muito os custos, já que foi possível obter grandes melhorias com o uso de processos ágeis, mantendo ainda a robustez, controle, estabilidade e previsibilidade de um processo CMMI nível 5.

\section{Referências}

Ambler, S., Jeffries, R. Agile Modeling: Effective Practices for Extreme Programming and the Unified Process. Wiley, 2002.

Beck, K. Extreme Programming Explained: Embrace Change. Addison-Wesley, 1999.

Beck, K. et. al. Manifesto for Agile Software Development, 2001.

Boehm, B. A View of 20th and 21st Century Software Engineering, ICSE, 2006.

Chrissis, M., Konrad, M., Shrum, S. CMMI Guidelines for Process Integration and Product Improvement. Second Edition, Addisson-Wesley, EUA, 2006.

Hehn, H. F. Peopleware: como trabalhar o fator humano nas implantações de sistemas. São Paulo: Gente, 2008.

Marçal, A. S et. al. Integração de Story Points e Use Case Points em Projetos Baseados em SCRUM e CMMI, SBQS 2009, Ouro Preto - MG, 2009.

Marçal, A. S. Scrummi: Um processo de gestão ágil baseado no SCRUM e aderente ao CMMI. Orientadora: Profa. Dra. Elizabeth Furtado. Dissertação de Mestrado. UNIFOR - Fortaleza, Brasil, 2009.

Schwaber, K. Agile Project Management With Scrum, Microsoft Press, Redmond, Washington, USA, 2004.

Tayntor, C. B. Six Sigma Software Development, Flórida, Auerbach, 2003.

Watson, G. H. Cycles of learning: observations of Jack Welck, ASQ Publication, 2007. 